\title{
Outcome of Percutaneous Transforaminal Endoscopic Lumbar Surgery in $>60$-Year-Old Patients with Low Back Pain
}

\author{
Ratish Singh, Gao Zeng Xin, Mangal Prasad Hirachan, Lin Yu Cheng \\ Department of Spine Surgery, Zhongda Hopital, Medical School of Southeast University, Nanjing, China
}

\begin{abstract}
Study Design: Retrospective study.
Purpose: To evaluate the outcome of percutaneous transforaminal endoscopic lumbar surgery (PTELS) and to evaluate the efficacy of this technique in the treatment of lumbar disc herniation (LDH) and lumbar spine stenosis (LSS) in >60-year-old patients.

Overview of Literature: There has been number of studies done in aspect of PTELS. All those studies focused on overall clinical aspect with no any age specific analysis. This articles deals with the patient $>60$ years old with low back pain (LBP) treated with the PTELS. This study provides the details of clinical outcome after the surgery in those age group.

Methods: We retrospectively studied 77 patients aged $>60$ years; out of these, 45 patients presented with LDH and 22 patients with lumbar spinal stenosis who underwent PTELS.

Results: The mean age of the patients who underwent the surgery was $68.33 \pm 6.97$ years and the mean duration of the surgery and the postoperative duration of hospitalization were $87.31 \pm 24.746$ minutes and $79 \pm 2.711$ days, respectively. The pre- and postoperative Oswestry Disability Index were $52.8022 \pm 11.98299$ and $16.3513 \pm 12.97398(p<0.05)$, respectively, indicating statistical significance. The pre- and postoperative visual analogue scale scores of the leg and back were $6.27 \pm 1.213$ and $4.79 \pm 1.162(p<0.05)$ and $1.40 \pm 1.688$ and $1.30 \pm 1.436$, respectively $(p<0.05)$, which showed a significant difference. Based on the MacNab criteria, $76.1 \%, 10 \%$, and $6 \%$ of the patients showed excellent or good, fair, and poor outcomes, respectively. Incomplete removal of content and reherniation were observed in $3 \%$ and $7.5 \%$ of the patients. No significant difference was found between the clinical outcomes of PTELS between LDH and LSS.

Conclusions: PTELS can be a good technique in spine surgery to relieve symptoms for LBP with satisfactory outcome, short duration of hospitalization, and advantages such as elimination of general anesthesia in elderly patients.
\end{abstract}

Keywords: Endoscopic surgery; Low back pain; Lumbar stenosis; Lumbar disc herniation; Visual Analogue Scale

\section{Introduction}

Low back pain (LBP) is one of the common reasons for hospital visit among the elders $[1,2]$. It is a common dis- order involving the muscles, nerves, and bones of the back $[3,4]$. Approximately $9 \%-12 \%$ of individuals can experience LBP in their lifetime, and approximately $25 \%$ individuals have reported its occurrence for a 1-month

\footnotetext{
Received Aug 8, 2017; Revised Sep 12, 2017; Accepted Oct 1, 2017

Corresponding author: Gao Zeng Xin

Department of Spine Surgery, Zhongda Hospital, School of Medicine, Southeast University, Nanjing, Jiangsu Province, China

Tel: +86-18912965566, Fax: +86-25-83324887, E-mail: gaozengxin@outlook.com
} 
period [5]. Approximately $70 \%-80 \%$ of the population experiences LBP with/without leg pain in their lifetime, and it is one of the causes for huge economic burden to families and the society [6]. LBP equally affects men and women [7], and it is more common at the ages $40-80$ years $[8,9]$. However, not all patients with back and leg pain require surgical treatment, but surgery is considered when the signs and symptoms progressively increase despite conservative treatment for $>6$ weeks [10]. Numerous surgical interventions for spinal surgeries, broadly classified as posterior open decompression and percutaneous techniques, are currently available [11]. Since its introduction 34 years prior, the percutaneous technique for spinal surgery has been practiced more worldwide because of its comparable significant outcome, minimal invasiveness, shorter duration of hospitalization, and elimination of general anesthesia $[12,13]$. The aim of this study was to evaluate the outcome of percutaneous endoscopic lumbar surgery in $>60$-year-old patients and to determine the efficacy of this technique in lumbar disc herniation (LDH) and lumbar spine stenosis (LSS) in these age group patients.

\section{Materials and Methods}

\section{Study design and methods}

We retrospectively followed up 77 patients aged $>60$ years with LDH and LSS who underwent percutaneous transforaminal endoscopic lumbar surgery (PTELS) in Zhongda Hospital affiliated Southeast University, Nanjing, China from June 2013 to June 2016. This is a retrospective study which does not require the Institutional Review Board approval. The study was done with informed consent to all included patients. The patients undergoing PTELS were selected based on the following: (1) failure of conservative treatment under medication and physical therapy for $>6$ weeks; (2) symptoms and their physical examination; and (3) radiological evaluation. Of the 77 patients, six died of natural causes and four did not follow-up. Hence, the remaining 67 patients were considered for the study. The follow-up was performed by telephone inquiry and during their visits to the outpatient department (OPD). Among these patients, 45 and 22 patients had LDH and LSS, respectively. The patients were diagnosed with $\mathrm{LDH}$ and LSS based on the clinical signs and symptoms, computed tomography (CT), and magnetic resonance imaging (MRI)
[14].

Self-evaluation questionnaires of the Oswestry Disability Index (ODI) and MacNab criteria were used for clinical outcomes [15]. The Visual Analogue Scale (VAS) was used to assess pain [16]. The evaluation of the recurrence of the symptoms, complications, postoperative duration of hospitalization, and duration of surgery was reviewed during the OPD visit and through telephone inquiries, and MRI, CT scans, and X-ray were performed in all patients.

IBM SPSS Statistics ver. 24.0 desktop (IBM Corp., Armonk, NY, USA) was used to perform statistical analysis. Paired $t$-test was used to determine pre- and postoperative ODI for all cases. Comparison of LDH and LSS was performed using independent sample $t$-test, non-parametric two independent sample tests, and Fisher's exact test. A $p$-value of $<0.05$ was considered to be statistically significant.

\section{Surgical technique for percutaneous transforaminal endoscopic lumbar surgery}

The patients were prepared for the surgery after assessing all preoperative evaluations. The patients were placed on the operation theater table in the prone or lateral decubitus position. If the patients were placed in the prone position, then the hip and knee were flexed and a pillow was placed between the lower abdomen and chest to permit free suspension of the abdomen (Fig. 1A). If the patients were placed in the lateral decubitus position, a pillow was placed under the waist, which would open the foramen and allow the dura to drop to the contralateral side that would prevent the damage on the introduction of the cannula and reduce intra-abdominal pressure and decrease bleeding. Thus, under the guidance of $\mathrm{c}$-arm entry point for surgery, 8-16 $\mathrm{cm}$ from the middle of the vertebra to the lateral was marked based on the disc interval that needed to be removed at the angle of $15^{\circ}-25^{\circ}$ in the horizontal plane [17]. Under aseptic conditions and local anesthesia ( $1 \%$ lidocaine), a 25 -cm 18 -gage needle was placed at the level of herniated disc via the posterolateral approach after identifying the anteroposterior and lateral views with the c-arm (Fig. 1B).

Subsequently, an intraoperative discogram with a 2-mL mixture of contrast medium and methylene blue at a 9:1 ratio was performed (Fig. 1C). A tear in annulus is indicated if the dye leaked in the epidural space [18]. Subsequently, the guide wire was inserted, and the needle 

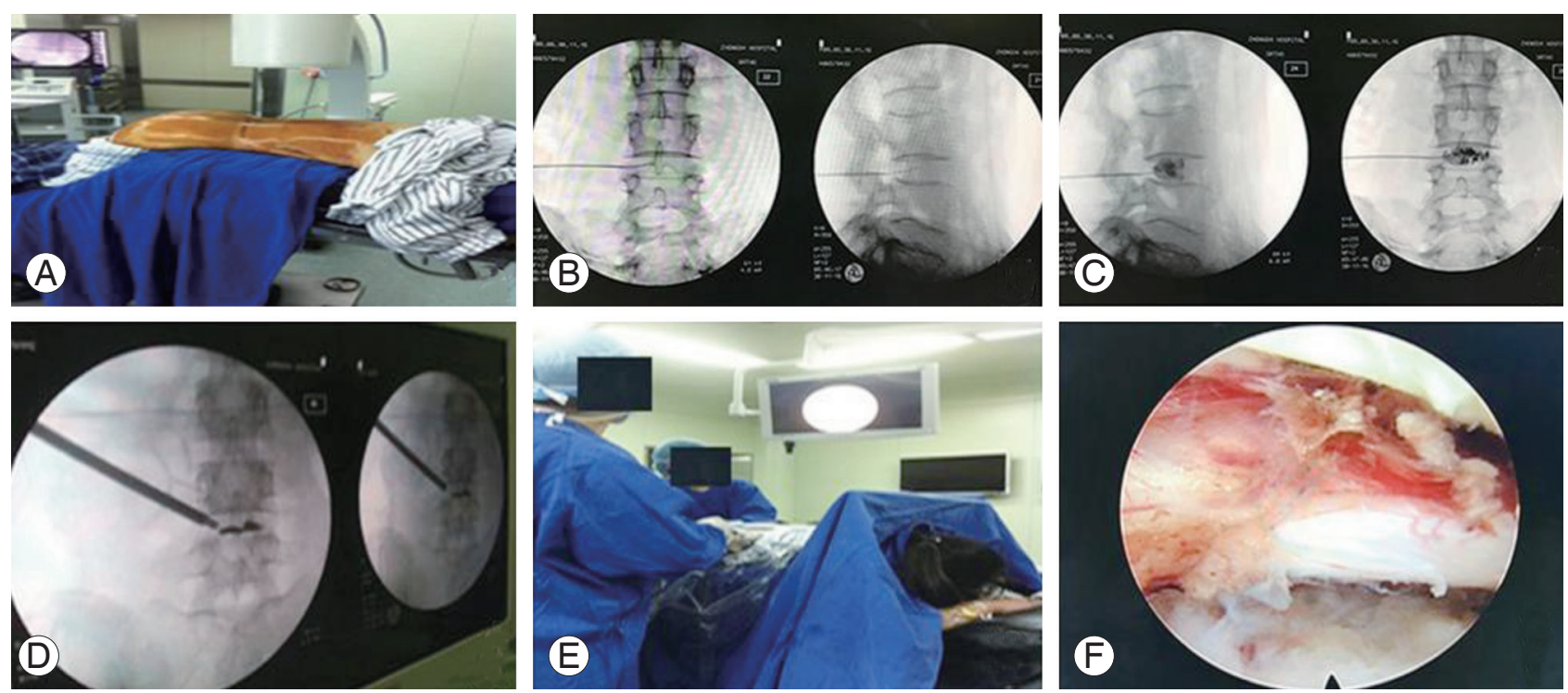

Fig. 1. Showing percutaneous transforaminal endoscopic lumbar surgery done at Zhongda Hospital affiliated to Southeast University. (A) Patient position for surgery. (B) Needle insertion. (C) Discogram with mixture of contrast medium and methylene blue. (D) Reaming over dilator. (E) Removal of content with endoscope. (F) Free nerve root after surgery.

Table 1. Pre- and postoperative ODI, VAS leg, and VAS back

\begin{tabular}{lccc} 
Variable & Preoperative & Postoperative & $p$-value \\
ODI & $52.8022 \pm 11.98299$ & $16.3513 \pm 12.97398$ & 0.000 \\
VAS leg & $6.27 \pm 1.213$ & $1.40 \pm 1.688$ & 0.000 \\
VAS back & $4.79 \pm 1.162$ & $1.30 \pm 1.436$ & 0.000 \\
\hline
\end{tabular}

Values are presented as mean \pm standard deviation.

ODI, Oswestry Disability Index; VAS, Visual Analogue Scale.

was removed. Furthermore, an 8-mm skin incision was constructed over the guide. A 2-mm conical dilating rod was introduced over the guide wire; then, the second and third sleeves dilating the soft tissues were sequentially introduced up to $6.5 \mathrm{~mm}$. A reamer was introduced counterclockwise to prevent damage to the spinal muscles. By continuously monitoring the image intensifier, the lamina was reamed and was safely advanced to 1 or $2 \mathrm{~mm}$ inside the medial pedicular line from the smaller to larger reamer (Fig. 1D). The working cannula was then introduced over the third conical rod. Its tip should be located on the herniated disc. Subsequently, the image intensifier was removed, and the working endoscope set was introduced to remove the content in the canal under direct visualization (Fig. 1E). After complete decompression, the dural sac and lumbar-exiting nerve root was checked for being freely movable with Valsalva maneuver (Fig. 1F). Bleeding of the small vessels was controlled using a flexible bipolar radio frequency probe. Communication with the patients was maintained throughout the procedure.

\section{Results}

\section{Clinical outcomes of percutaneous transforaminal endoscopic lumbar surgery in patients $>60$ years old}

The total number of patients evaluated was 67 (34 men and 33 women). The mean age of the patient who underwent surgery was $68.33 \pm 6.97$ years. Of the 67 patients, 45 and 22 patients had LDH and LSS, respectively. The mean follow-up period was 20.87 months. The mean duration of the surgery and postoperative duration of hospitalization were $87.31 \pm 24.746$ minutes and $3.79 \pm 2.711$ days, respectively. The pre- and postoperative ODI was $52.8022 \pm 11.98299$ and $16.3513 \pm 12.97398(p<0.05)$, indicating statistical significance (Table 1). The VAS scores of the leg and back pre- and postoperatively were $6.27 \pm 1.213$ and $4.79 \pm 1.162(p<0.05)$ and $1.40 \pm 1.688$ and $1.30 \pm 1.436$ 
Table 2. MacNab criteria

\begin{tabular}{lcccc} 
Outcome & Frequency & $\%$ & Valid $\%$ & Cumulative $\%$ \\
\hline Excellent & 27 & 40.3 & 40.3 & 40.3 \\
Good & 24 & 35.8 & 35.8 & 76.1 \\
\hline Fair & 10 & 14.9 & 14.9 & 91.0 \\
\hline Poor & 6 & 9.0 & 9.0 & 100.0 \\
Total & 67 & 100.0 & 100.0 & - \\
\hline
\end{tabular}

$(p<0.05)$, respectively, with significant difference (Table 1). Based on the MacNab criteria, $76.1 \%, 10 \%$, and $6 \%$ of the patients showed excellent or good, fair, and poor outcomes, respectively (Table 2). An incomplete removal of content and reherniation were observed in 3\% (2) and $7.5 \%$ (5) of the patients, respectively.

\section{Comparative study of clinical outcomes of percuta- neous transforaminal endoscopic lumbar surgery between lumbar disc herniation and lumbar spine stenosis}

The clinical outcome of PTELS between LDH and LSS was compared based on ODI, VAS scores of the leg and back, MacNab criteria, duration of surgery, postoperative duration of hospitalization, and complications. Forty-five patients had LDH (26 men and 19 women), whereas 22 patients had LSS (eight men and 14 women). The mean age of the patients with LDH and LSS was 66.84 \pm 6.098 years and $71.36 \pm 9.302$ years, respectively. The duration of surgery in the two groups was $87.67 \pm 25.486$ and $86.59 \pm 23.724$ minutes $(p=0.537$ ), respectively, showing no significant difference between the two groups. The postoperative duration of hospitalization was $3.09 \pm 3.088$ and $2.18 \pm 1.593$ days $(p=0.484)$, respectively. No statistically significance difference was found between LDH and LSS based on ODI (36.7276 \pm 19.16158 and $35.8850 \pm 17.70137$, respectively; $p=0.863$ ) (Table 3 ). No significant difference was found between the two groups in terms of the VAS scores of the leg and back $(p=0.193$ and $p=0.897$, respectively). No statistically significant difference was found based on the MacNab criteria $(p=0.090)$. Of the 45 patients with $\mathrm{LDH}$, four $(8.9 \%)$ had reherniation. Of the 22 patients with LSS, one (4.5\%) and two (9.1\%) had of reherniation and incomplete removal of content, respectively. Based on the MacNab criteria, the outcome was $48.9 \%$ excellent, $28.9 \%$ good, $15.6 \%$ fair, and $6.7 \%$ poor in
Table 3. ODI of patients in the LDH and LSS groups

\begin{tabular}{cccc} 
& LDH & LSS & $p$-value \\
ODI & $-36.7276 \pm 19.16158$ & $-35.8850 \pm 17.70137$ & 0.863 \\
\hline
\end{tabular}

Values are presented as mean \pm standard deviation.

ODI, Oswestry Disability Index; LDH, lumbar disc herniation; LSS, lumbar spine stenosis.

Table 4. MacNab score of patients in the LDH and LSS groups

\begin{tabular}{lcccc} 
Group & Excellent & Good & Fair & Poor \\
LDH & 22 & 13 & 7 & 3 \\
LSS & 5 & 11 & 3 & 3 \\
Total & 27 & 24 & 10 & 6 \\
\hline
\end{tabular}

$\mathrm{LDH}$, lumbar disc herniation; LSS, lumbar spine stenosis.

the LDH group and $22.2 \%$ excellent, $50.0 \%$ good, $13.6 \%$ fair, and $13.6 \%$ poor in the LSS group (Table 4).

\section{Discussion}

Percutaneous endoscopic surgery is one of the latest emerging techniques in the treatment of LDH [18]. After the introduction of non-visualized percutaneous central nucleotomy via posterolateral approach by Kambin and Gellman [19] in 1973 and Hijikata [20] in 1975 and the introduction of direct visualization of intervertebral disc space with modified arthroscope by Forst and Hausmann [21] in 1983, it has been a widely practiced technique for spinal surgery thereafter. Two techniques are recently widely used for percutaneous endoscopic surgery: (1) the transforaminal approach described by Yeung et al. [22] (Yeung endoscopic spine system technique) in 1997 and Hoogland et al. [23] (transforaminal endoscopic surgery system) in 1994 and (2) the interlaminar approach described by Ruetten et al. [24] in 2007. After the development of day-care surgery concept, minimal invasive 


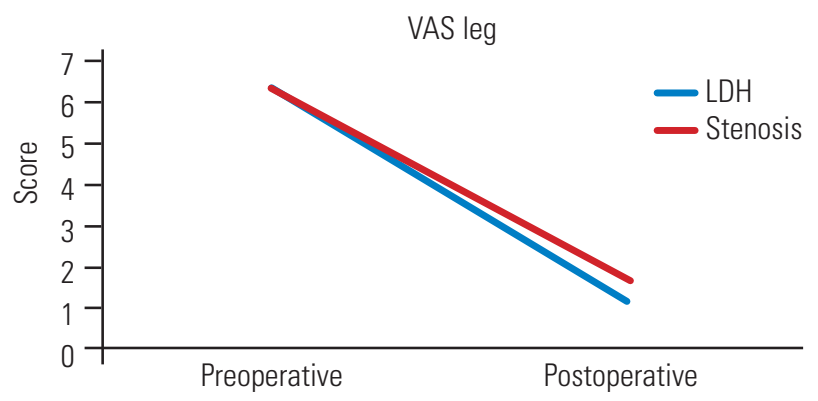

Fig. 2. Graphic comparison of pre- and postoperative VAS of leg in LDH and stenosis. VAS, Visual Analogue Scale; LDH, lumbar disc herniation.

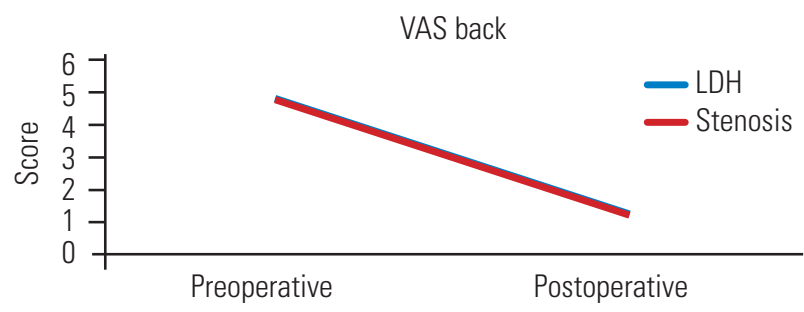

Fig. 3. Graphic comparison of pre- and postoperative VAS of back in LDH and stenosis. VAS, Visual Analogue Scale; LDH, lumbar disc herniation.

technique, and the idea of eliminating general anesthesia, patients are more interested in undergoing PELS. Based on the ODI, VAS, MacNab criteria, and postoperative duration of hospitalization, PTELS has shown a good clinical outcome compared with that by the traditional open lumbar discectomy, microdiscectomy, and other techniques.

Based on the MacNab criteria, a study conducted in Taiwan showed that $89 \%$ of patients postoperatively achieved excellent (28\%) or good (61\%) outcomes [25]. To date, different studies showed that $80 \%-96.7 \%$ patients had good outcome after PTELS [24,26,27]. Li et al. [28] reported a case series of 85 patients with lumbar lateral recess stenosis with/without combined herniated discs treated with percutaneous lumbar foraminoplasty-percutaneous endoscopic lumbar discectomy; 90.6\% patients had excellent or good outcome based on the MacNab criteria. In our study, $76.1 \%$ of the patients had an excellent or good outcome based on the MacNab criteria, which was comparatively lower than the result from the studies mentioned above. We presumed that the difference might be due to old age, few samples, and other comorbid conditions of the patients. Furthermore, the results of the previous study are from young adults. No postoperative significant difference was found in the clinical outcomes between LDH and LSS in these age group patients. In this study, the postoperative ODI improved, which was similar and consistent with that of the other studies to date. Our study on LSS in 2014 showed that the ODI at 1 and 24 months postoperatively were better than that preoperatively in the two groups $(p<0.05)$, and the improvement of ODI in the PTELS group was also better than that in the traditional surgery group $(p<0.05)$. The VAS score of the leg and back pain decreased from 7.5 to 1.7 and from 6.0 to 2.3 , respectively. Our study also emphasized that the pre- and postoperative VAS score of the leg and back significantly improved, whereas the postoperative VAS score of the leg and back between LDH and LSS was not significantly different ( $p=0.193$ and $p=0.897$, respectively) (Figs. 2, 3). In elderly patients, the improvement of VAS in the PTELS group was better than that in the traditional surgery group $(p<0.05)$ [29].

Although the surgical treatment may be more effective, there is always small risk of developing complications [30]. A study also showed that $1 \%-2 \%$ and $1 \%-2 \%$ of patients had dural tear and nerve injury, respectively. In our study, no significant complication, such as dural tear, dysesthesia, and nerve root injuries were observed. Other studies recently showed that $3 \%-6.6 \%$ had reherniation $[17,24,27,28]$. In our study $3 \%$ (two cases) of incomplete removal of content and 7.5\% (five cases) reherniation were seen after the surgery. Of the 45 patients with LDH, four (8.9\%) had reherniation. Of the 22 patients with LSS, one (4.5\%) and two $(9.1 \%)$ had of reherniation and incomplete removal of content, respectively. Five patients with reherniation underwent further fusion surgery after a few years. Furthermore, revised PTELS was performed after 1 month of the first surgery in the patients with incomplete removal of contents. PTELS is safe for the treatment of the patients with LDH and LSS, which was completed in an hour, with rapid postoperative recovery. These clinical outcomes suggested that PTELS is applicable for patients $>60$ years of age with LDH and LSS.

\section{Conclusions}

The indications for transforaminal endoscopic treatment are the same as those of classical discectomy. Due to the advancement of instrumentation and surgeon skills, the indication has currently broadened. Our study showed that no significant difference was found between the clinical outcomes of PTELS between LDH and LSS in patients $>60$ years. Currently, PTELS can be an alternative tech- 
nique in spine surgery with satisfactory outcome, short duration of hospitalization, and elimination of general anesthesia for elderly patients who cannot usually tolerate general anesthesia, significantly benefiting them from the PTELS surgery. Although different studies show satisfactory clinical outcome, further studies are warranted.

\section{Conflict of Interest}

No potential conflict of interest relevant to this article was reported.

\section{References}

1. Mantyselka P, Kumpusalo E, Ahonen R, et al. Pain as a reason to visit the doctor: a study in Finnish primary health care. Pain 2001;89:175-80.

2. Hart LG, Deyo RA, Cherkin DC. Physician office visits for low back pain: frequency, clinical evaluation, and treatment patterns from a U.S. national survey. Spine (Phila Pa 1976) 1995;20:11-9.

3. Panjabi MM. A hypothesis of chronic back pain: ligament subfailure injuries lead to muscle control dysfunction. Eur Spine J 2006;15:668-76.

4. Nachemson AL. Newest knowledge of low back pain: a critical look. Clin Orthop Relat Res 1992;(279):820.

5. Hoy D, Brooks P, Blyth F, Buchbinder R. The epidemiology of low back pain. Best Pract Res Clin Rheumatol 2010;24:769-81.

6. Parthan A, Evans CJ, Le K. Chronic low back pain: epidemiology, economic burden and patient-reported outcomes in the USA. Expert Rev Pharmacoecon Outcomes Res 2006;6:359-69.

7. Perennou D, Marcelli C, Herisson C, Simon L. Adult lumbar scoliosis: epidemiologic aspects in a low-back pain population. Spine (Phila Pa 1976) 1994;19:1238.

8. Svensson HO, Andersson GB. The relationship of low-back pain, work history, work environment, and stress: a retrospective cross-sectional study of 38- to 64-year-old women. Spine (Phila Pa 1976) 1989;14:517-22.

9. Hartvigsen J, Christensen K, Frederiksen H. Back pain remains a common symptom in old age: a population-based study of 4486 Danish twins aged 70102. Eur Spine J 2003;12:528-34.
10. Waddell G. 1987 Volvo award in clinical sciences: a new clinical model for the treatment of low-back pain. Spine (Phila Pa 1976) 1987;12:632-44.

11. Sinkemani A, Hong X, Gao ZX, et al. Outcomes of microendoscopic discectomy and percutaneous transforaminal endoscopic discectomy for the treatment of lumbar disc herniation: a comparative retrospective study. Asian Spine J 2015;9:833-40.

12. Postacchini F, Postacchini R. Operative management of lumbar disc herniation. In: Alexandre A, Masini M, Menchetti PP, editors. Advances in minimally invasive surgery and therapy for spine and nerves. Wien: Springer-Verlag; 2011. p.17-21.

13. Hirachan MP, Gao Z, Lin Y, Singh R. Clinical outcome of percutaneous endoscopic lumbar surgery (PELS) in treatment of lumbar disc herniation. Open J Orthop 2017;7:99.

14. Inui Y, Doita M, Ouchi K, Tsukuda M, Fujita N, Kurosaka $\mathrm{M}$. Clinical and radiologic features of lumbar spinal stenosis and disc herniation with neuropathic bladder. Spine (Phila Pa 1976) 2004;29:869-73.

15. Smith JS, Ogden AT, Shafizadeh S, Fessler RG. Clinical outcomes after microendoscopic discectomy for recurrent lumbar disc herniation. J Spinal Disord Tech 2010;23:30-4.

16. Carlsson AM. Assessment of chronic pain: I. aspects of the reliability and validity of the visual analogue scale. Pain 1983;16:87-101.

17. Ahn SS, Kim SH, Kim DW, Lee BH. Comparison of outcomes of percutaneous endoscopic lumbar discectomy and open lumbar microdiscectomy for young adults: a retrospective matched cohort study. World Neurosurg 2016;86:250-8.

18. Mayer HM, Brock M. Percutaneous endoscopic discectomy: surgical technique and preliminary results compared to microsurgical discectomy. J Neurosurg 1993;78:216-25.

19. Kambin P, Gellman H. Percutaneous lateral discectomy of the lumbar spine: a preliminary report. Clin Orthop Relat Res 1983;174:127-32.

20. Hijikata S. Percutaneous nucleotomy: a new concept technique and 12 years' experience. Clin Orthop Relat Res 1989;(238):9-23.

21. Forst R, Hausmann B. Nucleoscopy: a new examination technique. Arch Orthop Trauma Surg 1983;101:219-21.

22. Yeung AT, Tsou PM. Posterolateral endoscopic exci- 
sion for lumbar disc herniation: surgical technique, outcome, and complications in 307 consecutive cases. Spine (Phila Pa 1976) 2002;27:722-31.

23. Hoogland T, Schubert M, Miklitz B, Ramirez A. Transforaminal posterolateral endoscopic discectomy with or without the combination of a lowdose chymopapain: a prospective randomized study in 280 consecutive cases. Spine (Phila Pa 1976) 2006;31:E890-7.

24. Ruetten S, Komp M, Merk H, Godolias G. Fullendoscopic interlaminar and transforaminal lumbar discectomy versus conventional microsurgical technique: a prospective, randomized, controlled study. Spine (Phila Pa 1976) 2008;33:931-9.

25. Tzaan WC. Transforaminal percutaneous endoscopic lumbar discectomy. Chang Gung Med J 2007;30:22634.

26. Lee JS, Kim HS, Jang JS, Jang IT. Structural preservation percutaneous endoscopic lumbar interlaminar discectomy for L5-S1 herniated nucleus pulposus. Biomed Res Int 2016;2016:6250247.
27. Ahn Y, Lee SH, Park WM, Lee HY, Shin SW, Kang HY. Percutaneous endoscopic lumbar discectomy for recurrent disc herniation: surgical technique, outcome, and prognostic factors of 43 consecutive cases. Spine (Phila Pa 1976) 2004;29:E326-32.

28. Li ZZ, Hou SX, Shang WL, Cao Z, Zhao HL. Percutaneous lumbar foraminoplasty and percutaneous endoscopic lumbar decompression for lateral recess stenosis through transforaminal approach: technique notes and 2 years follow-up. Clin Neurol Neurosurg 2016;143:90-4.

29. Hu DX, Zheng Q, Zhu B, Ying XZ, Wang YF. Percutaneous intervertebral foramina endoscopic lumbar discectomy decompression for elder patients with lumbar spinal stenosis syndrome. Zhongguo Gu Shang 2014;27:194-8.

30. Pan L, Zhang P, Yin Q. Comparison of tissue damages caused by endoscopic lumbar discectomy and traditional lumbar discectomy: a randomised controlled trial. Int J Surg 2014;12:534-7. 